\title{
Properties of interval-valued intuitionistic fuzzy vector spaces
}

\author{
R. Santhi ${ }^{1}$ and N. Udhayarani ${ }^{2}$ \\ ${ }^{1}$ PG and Research Department of Mathematics, Nallamuthu Gounder Mahalingam College \\ Pollachi, Tamil Nadu, 642001 India \\ e-mail: santhifuzzy@yahoo.co.in \\ 2 Department of Mathematics, Nallamuthu Gounder Mahalingam College \\ Pollachi, Tamil Nadu, 642001 India \\ e-mail: udhayaranin@gmail.com
}

\begin{abstract}
In this paper we introduced and studied the properties of interval-valued intuitionistic fuzzy vector spaces (in brief IVIF space) and its IVIF standard basis. We use the concept of max-union and min-intersection algebra to define interval-valued ituitionistic fuzzy vector space.
\end{abstract} Keywords: Interval-valued intuitionistic fuzzy vector space, IVIF subspace, IVIF span, IVIF spanning set, $\operatorname{dim}(\widetilde{S})$, IVIF basis, IVIF standard basis.

2010 Mathematics Subject Classification: 46A40, 52A07.

\section{Introduction}

The concept of fuzzy set was introduced by L. A. Zadeh [16]. Basic definition and propositions of interval-valued fuzzy vector space is introduced by S. Mondal [11]. In 1977, A. K. Katsaras and D. B. Liu [7], apply the concept of fuzzy set theory in elementary theory of vector spaces and introduced fuzzy vector spaces and fuzzy topological vector spaces. In 1983, K. Atanassov extended the notion of fuzzy sets to intuitionistic fuzzy sets [1]. In 2017, M. Chiney and S. K. Samanta [3], introduced a notion of intuitionistic fuzzy vector space and intuitionistic fuzzy basis. R. Pradhan and M. Pal [13,14], discussed about intuitionistic fuzzy linear transformations. 
Also they developed a system of intuitionistic fuzzy linear equations and tolerable solution of an unsolved system. In 2007, S. B. Hosseini et al. [5], defined the concept of intuitionistic fuzzy metric and normed spaces and prove several theorms about completeness and compactness. Using level subsets and image $\mu, \mathrm{R}$. Kumar [8], redefined fuzzy subspaces and fuzzy cosets.

In 1991, D.S. Malik and J.N. Mordeson [10] characterize the conditions about fuzzy subspace of a vector space and introduce the concept of fuzzy freeness of a fuzzy subset of a vector space. In 1993, J.N.Mordeson [12] gives the conditions to have a basis over a fuzzy subfield. In 1990, P.Lubzonok [9] defined the fuzzy dimension for all fuzzy vector spaces as a non negative real number or infinity and proved some theorems based on fuzzy finite dimension in vector spaces. F.G.Shi and C.E. Huang [15] redefine the fuzzy dimension of fuzzy vector spaces. In 2012, the same authors [6] consider the direct sum of fuzzy dimension of fuzzy vector spaces. In 2001, M. Gehrke et al. [4] concerned with the basics of a theory for such 'interval-valued' fuzzy sets. Using lattice theory, they build the concept of the interval-valued fuzzy set theory.

\section{Preliminaries}

The following preliminary concepts are studied to develop the basic properties of interval-valued intuitionistic fuzzy vector spaces.

Definition 2.1. Interval-valued Fuzzy Set [11]: An interval-valued fuzzy set (IVFS) A on the universe $U \neq \phi$ is given by $A=\{(u, A(u)): u \in U\}$, where $A(u)=[\underline{A}(u), \bar{A}(u)] \in L([0,1])$ being $L([0,1])=\left\{x=[\underline{x}, \bar{x}]:[\underline{x}, \bar{x}] \in[0,1]^{2}\right.$ and $\left.\underline{x} \leq \bar{x}\right\}$.

Obviously, $A(u)=[\underline{A}(u), \bar{A}(u)]$ is the membership degree and $\underline{A}(u), \bar{A}(u)$ are the lower and the upper limits of the membership degree of $u \in U$. Let I be the set of all real numbers lying between 0 and 1. That is, $I=\{x: 0 \leq x \leq 1\}$. Also let $D[0,1]$ be the interval $[0,1]$ which can be written as $D=\{[a, b]: a \leq b ; a, b \in I\}$.

Definition 2.2. Interval-valued fuzzy vector [11]: An interval-valued fuzzy vector is an n-tuple of elements from an interval-valued fuzzy algebra. That is, an IVFV is of the form $\left(x_{1}, x_{2}, \ldots, x_{n}\right)$, where each element $x_{i} \in F, i=1,2, \ldots, n$.

Definition 2.3. Interval-valued fuzzy vector space [11]: An interval-valued fuzzy vector space (IVFVS) is a pair $(E, A(x))$ where $E$ is a vector space in crisp sense and $A: E \rightarrow D[0,1]$ with the property, that for all $a, b \in F$ and $x, y \in E$, we have $\underline{A}(a x+b y) \geq \underline{A}(x) \wedge \underline{A}(y)$ and $\bar{A}(a x+b y) \geq \bar{A}(x) \wedge \bar{A}(y)$.

Definition 2.4. Interval-valued intuitionistic fuzzy set [2]: Let $D[0,1]$ be the set of closed subintervals of the interval $[0,1]$ and $X(\neq \phi)$ be a given set. An interval-valued intuitionistic fuzzy set in $X$ is defined as, $A=\left\{\left\langle x, \mu_{A}(x), \nu_{A}(x)\right\rangle \mid x \in X\right\}$, where $\mu_{A}: X \rightarrow D[0,1], \nu_{A}: X \rightarrow D[0,1]$ with the condition $0 \leq \sup \left(\mu_{A}(x)\right)+\sup \left(\nu_{A}(x)\right) \leq 1$ for any $x \in X$. The intervals $\mu_{A}(x)$ and $\nu_{A}(x)$ denotes the degree of belongingness and the degree of nonbelongingness of the element $x$ 
to the set $A$. Thus for each $x \in X, \mu_{A}(x)$ and $\nu_{A}(x)$ are closed intervals and their lower and upper end points are denoted by $\mu_{A_{L}}(x), \mu_{A_{U}}(x), \nu_{A_{L}}(x)$ and $\nu_{A_{U}}(x)$. We can denote by:

$$
A=\left\{\left\langle x,\left[\mu_{A_{L}}(x), \mu_{A_{U}}(x)\right],\left[\nu_{A_{L}}(x), \nu_{A_{U}}(x)\right]\right\rangle \mid x \in X\right\},
$$

where $0 \leq \mu_{A_{U}}(x)+\nu_{A_{U}}(x) \leq 1, \mu_{A_{L}}(x) \geq 0, \nu_{A_{L}}(x) \geq 0$. For each element $x$ we can compute the unknown degree (hesitancy degree) of an intuitionistic fuzzy interval of $x \in X$ in $A$ defined as follows:

$$
\pi_{A}(x)=1-\mu_{A}(x)-\nu_{A}(x)=\left[1-\mu_{A_{U}}(x)-\nu_{A_{U}}(x), 1-\mu_{A_{L}}(x)-\nu_{A_{L}}(x)\right]
$$

Especially, if $\mu_{A}(x)=\mu_{A_{U}}(x)=\mu_{A_{L}}(x)$ and $\nu_{A}(x)=\nu_{A_{U}}(x)=\nu_{A_{L}}(x)$, then the given IVIFS A is reduced to an ordinary intuitionistic fuzzy set. For two IVIFSs

$$
A=\left\{\left\langle x,\left[\mu_{A_{L}}(x), \mu_{A_{U}}(x)\right],\left[\nu_{A_{L}}(x), \nu_{A_{U}}(x)\right]\right\rangle \mid x \in X\right\}
$$

and

$$
B=\left\{\left\langle x,\left[\mu_{B_{L}}(x), \mu_{B_{U}}(x)\right],\left[\nu_{B_{L}}(x), \nu_{B_{U}}(x)\right]\right\rangle \mid x \in X\right\}
$$

the following two relations are defined:

1. $A \subseteq B$ if and only if

(a) $\mu_{A_{U}}(x) \leq \mu_{B_{U}}(x)$,

(b) $\mu_{A_{L}}(x) \leq \mu_{B_{L}}(x)$,

(c) $\nu_{A_{U}}(x) \geq \nu_{B_{U}}(x)$,

(d) $\nu_{A_{L}}(x) \geq \nu_{B_{L}}(x)$, for any $x \in X$.

2. $A=B$ if and only if

(a) $\mu_{A_{U}}(x)=\mu_{B_{U}}(x)$,

(b) $\mu_{A_{L}}(x)=\mu_{B_{L}}(x)$,

(c) $\nu_{A_{U}}(x)=\nu_{B_{U}}(x)$,

(d) $\nu_{A_{L}}(x)=\nu_{B_{L}}(x)$, for any $x \in X$.

\section{Interval-valued intuitionistic fuzzy vector space}

In this section, we introduce and study the concept of interval-valued intuitionistic fuzzy vector space and its properties.

Definition 3.1. Interval-valued Intuitionistic Fuzzy Vector Space: The mathematical system of interval-valued intuitionistic fuzzy algebra is defined with two binary operations “+” and “.” on the set $\widetilde{V}$, satisfying the following properties:

Let $x_{L}, y_{L}$ and $x_{U}, y_{U}$ be the lower and upper end points of membership and non-membership degrees, respectively. 
1. Idempotence : $\left[x_{L}, x_{U}\right]+\left[x_{L}, x_{U}\right]=\max \left\{\left[x_{L}, x_{U}\right],\left[x_{L}, x_{U}\right]\right\}=\left[x_{L}, x_{U}\right]$

2. Commutativity: $\left[x_{L}, x_{U}\right]+\left[y_{L}, y_{U}\right]=\left[y_{L}, y_{U}\right]+\left[x_{L}, x_{U}\right]$

3. Associativity: $\left[x_{L}, x_{U}\right]+\left(\left[y_{L}, y_{U}\right]+\left[z_{L}, z_{U}\right]\right)=\left(\left[x_{L}, x_{U}\right]+\left[y_{L}, y_{U}\right]\right)+\left[z_{L}, z_{U}\right]$

4. Absorption :

(a) $\left[x_{L}, x_{U}\right]+\left(\left[x_{L}, x_{U}\right] \cdot\left[y_{L}, y_{U}\right]\right)=\left[x_{L}, x_{U}\right]$,

(b) $\left[x_{L}, x_{U}\right] \cdot\left(\left[x_{L}, x_{U}\right]+\left[y_{L}, y_{U}\right]\right)=\left[x_{L}, x_{U}\right]$.

5. Universal bounds:

(a) $\left[x_{L}, x_{U}\right]+\phi=\left[x_{L}, x_{U}\right]$,

(b) $\left[x_{L}, x_{U}\right]+I=I$,

(c) $\left[x_{L}, x_{U}\right] \cdot \phi=\phi$,

(d) $\left[x_{L}, x_{U}\right] . I=\left[x_{L}, x_{U}\right]$.

where $\phi=\langle[0,0],[1,1]\rangle$ is the zero element and $I=\langle[1,1],[0,0]\rangle$ is the identity element. With these properties, we can define an interval-valued intuitionistic fuzzy vector space. The triplet $\left(V,\left[\mu_{L}(x), \mu_{U}(x)\right],\left[\nu_{L}(x), \nu_{U}(x)\right]\right)=\widetilde{V}$ is called interval-valued intuitionistic fuzzy vector space where $V$ is a vector space and $\alpha_{\mu_{U}}: \widetilde{V} \rightarrow D[0,1], \alpha_{\nu_{U}}: \widetilde{V} \rightarrow D[0,1], \mu_{L} \geq 0$ and $\nu_{L} \geq 0$ with the property that for all $\alpha, \beta \in \widetilde{V}$ and $x, y \in F$, then

1. $\left[\left(\alpha_{\mu_{L_{1}}}+\beta_{\mu_{L_{1}}}\right),\left(\alpha_{\mu_{U_{1}}}+\beta_{\mu_{U_{1}}}\right)\right] \in \widetilde{V}$

2. $\left[\left(\alpha_{\nu_{L_{1}}}+\beta_{\nu_{L_{1}}}\right),\left(\alpha_{\nu_{U_{1}}}+\beta_{\nu_{U_{1}}}\right)\right] \in \widetilde{V}$

3. $\left\{\left\langle\left[\left(\left(\alpha_{L} \wedge \mu_{L}\right),\left(\alpha_{U} \wedge \mu_{U}\right)\right),\left(\left(\left(1-\alpha_{L}\right) \vee \nu_{L},\left(1-\alpha_{U}\right) \vee \nu_{U}\right)\right)\right]\right\rangle\right\} \in \widetilde{V}$

where

$$
\begin{gathered}
\mu_{L_{1}}+\mu_{L_{2}}=\mu_{L_{1}} \vee \mu_{L_{2}}, \\
\mu_{U_{1}}+\mu_{U_{2}}=\mu_{U_{1}} \vee \mu_{U_{2}}, \\
\nu_{L_{1}}+\nu_{L_{2}}=\nu_{L_{1}} \wedge \nu_{L_{2}}, \\
\nu_{U_{1}}+\nu_{U_{2}}=\nu_{U_{1}} \wedge \nu_{U_{2}} .
\end{gathered}
$$

Example 3.2. Let $\widetilde{V}_{3}$ be the set of triplet over F. For $\alpha=\left\{\alpha_{1}, \alpha_{2}, \alpha_{3}\right\}$ and $\beta=\left\{\beta_{1}, \beta_{2}, \beta_{3}\right\}$. Let $A_{1}=\left\{\left\langle\alpha_{1},[0.1,0.2],[0.5,0.6]\right\rangle,\left\langle\alpha_{2},[0.1,0.2],[0.7,0.6]\right\rangle,\left\langle\alpha_{3},[0.5,0.6],[0.3,0.4]\right\rangle\right\}$ and $A_{2}=$ $\left\{\left\langle\beta_{1},[0.5,0.6],[0.3,0.4]\right\rangle,\left\langle\beta_{2},[0.3,0.4],[0.7,0.6]\right\rangle,\left\langle\beta_{3},[1,1],[0,0]\right\rangle\right\}$. Here $A_{1}+A_{2} \in \widetilde{V}_{3}$ and $x A_{1} \in \widetilde{V}_{3}$ where $x=\{\langle x,[0.5,0.6],[0.3,0.4]\rangle\}$. Therefore, $\widetilde{V}_{3}$ is an interval-valued intuitionistic fuzzy vector space.

Definition 3.3. Transpose: Let $\widetilde{V}^{t}=\left\{\left[\alpha_{L}, \alpha_{U}\right]^{t} /\left[\alpha_{L}, \alpha_{U}\right] \in \widetilde{V}\right\}$ where $\left[\alpha_{L}, \alpha_{U}\right]^{t}$ is the transpose of an interval-valued intuitionistic fuzzy vector $\left[\alpha_{L}, \alpha_{U}\right]$

For $\left[\alpha_{L}, \alpha_{U}\right],\left[\beta_{L}, \beta_{U}\right] \in \widetilde{V}^{t}$ and $\left[x_{L}, x_{U}\right] \in F$, then 
1. $\left(\left[x_{L}, x_{U}\right] \cdot\left[\alpha_{L}, \alpha_{U}\right]^{t}\right)^{t}=\left[x_{L}, x_{U}\right] \cdot\left[\alpha_{L}, \alpha_{U}\right]$,

2. $\left(\left[\alpha_{L}, \alpha_{U}\right]^{t}+\left[\beta_{L}, \beta_{U}\right]^{t}\right)^{t}=\left[\alpha_{L}, \alpha_{U}\right]+\left[\beta_{L}, \beta_{U}\right]$.

Definition 3.4. IVIF Subspace: Let $\widetilde{W}$ be a subset of $\widetilde{V}$ and $\left[\phi_{L}, \phi_{U}\right],\left[I_{L}, I_{U}\right] \in \widetilde{W}$. A subset $\widetilde{W}$ of $\widetilde{V}$ is said to be an IVIF subspace of $\widetilde{V}$, if for any $\left[\alpha_{L}, \alpha_{U}\right],\left[\beta_{L}, \beta_{U}\right] \in \widetilde{W}$ and $\left[\alpha_{L}, \alpha_{U}\right]+$ $\left[\beta_{L}, \beta_{U}\right] \in \widetilde{W}$.

Definition 3.5. IVIF Span of $\widetilde{S}:$ A linear combination of elements of the interval-valued intuitionistic fuzzy set of interval-valued intuitionistic fuzzy vector $\widetilde{S}$ is a finite sum $\sum\left[x_{L_{i}}, x_{U_{i}}\right]$ $\left[\alpha_{L_{i}}, \alpha_{U_{i}}\right]$ where $\left[x_{L_{i}}, x_{U_{i}}\right]$ and $\left[\alpha_{L_{i}}, \alpha_{U_{i}}\right] \in I$. The set of all linear combinations of $\widetilde{S}$ is called the IVIF span of $\widetilde{S}$ and is denoted by $\langle\widetilde{S}\rangle$.

Definition 3.6. IVIF spanning set: Let $\widetilde{S}$ and $\widetilde{W}$ be two intuitionistic fuzzy subsets of $\widetilde{V}$. If $\langle\widetilde{S}\rangle=\widetilde{W}$, then $\widetilde{S}$ is called an IVIF spanning set or set of IVIF generators for $\widetilde{W}$.

If $\widetilde{W}$ is an IVIF intuitionistic fuzzy subspace of $\widetilde{V}$ then $\langle\widetilde{W}\rangle=\widetilde{W}$.

Definition 3.7. IVIF Basis: An IVIF spanning set for $\widetilde{W}$ is called an IVIF basis for an IVIF subspace $\widetilde{W}$ of $\widetilde{V}$ which one have minimum cardinality.

Definition 3.8. Linearly dependence: An IVIF set $\widetilde{S}$ of vectors over an interval-valued intuitionistic fuzzy algebra $F$, is linearly dependent if at least one element of $\widetilde{S}$ is a linear combination of the other element of $\widetilde{S}$. Otherwise, it is called linearly independent.

Proposition 3.9. Let $A$ and $B$ be the set of interval-valued intuitionistic fuzzy vectors.

1. The set consisting of the zero vector is linearly dependent.

2. If $A \subset B$ and if $A$ is linearly dependent, then $B$ is also linearly dependent.

3. If $A \subset B$ and if $B$ is linearly independent, then $A$ is also linearly independent.

Proof. To prove (1): Let $\widetilde{S}=\left\{\alpha_{1}, \alpha_{2}, \ldots, \alpha_{r-1}, \phi, \alpha_{r+1}, \ldots, \alpha_{n}\right\}$, where $\alpha_{i}^{\prime} s$ are interval-valued intuitionistic fuzzy vectors for all $i \in\{1,2, \ldots, r-1, r+1, \ldots, n\}$ and $\phi$ be the interval-valued intuitionistic fuzzy zero vector. It can be written as

$$
\begin{aligned}
\phi & =\phi \cdot \alpha_{1}+\phi \cdot \alpha_{2}+\cdots+\phi \cdot \alpha_{r+1}+\cdots+\phi \cdot \alpha_{n} \\
& =\left\langle[(0,0),(1,1)] \cdot\left[\left(\mu_{L_{1}}, \mu_{U_{1}}\right),\left(\nu_{L_{1}}, \nu_{U_{1}}\right)\right]\right\rangle+\left\langle[(0,0),(1,1)] \cdot\left[\left(\mu_{L_{2}}, \mu_{U_{2}}\right),\left(\nu_{L_{2}}, \nu_{U_{2}}\right)\right]\right\rangle+\ldots \\
& +\left\langle[(0,0),(1,1)] \cdot\left[\left(\mu_{L_{n}}, \mu_{U_{n}}\right),\left(\nu_{L_{n}}, \nu_{U_{n}}\right)\right]\right\rangle \\
& =\langle[(0,0),(1,1)]\rangle .
\end{aligned}
$$

Hence $\phi$ is a linear combination of the other vectors in $S$. Thus $S$ is linearly dependent.

To prove (2): Let $A=\left\{\left[\alpha_{L_{1}}, \alpha_{U_{1}}\right],\left[\alpha_{L_{2}}, \alpha_{U_{2}}\right], \ldots,\left[\alpha_{L_{r}}, \alpha_{U_{r}}\right]\right\}$ and $B=\left\{\left[\alpha_{L_{1}}, \alpha_{U_{1}}\right],\left[\alpha_{L_{2}}, \alpha_{U_{2}}\right], \ldots\right.$, $\left.\left[\alpha_{L_{r}}, \alpha_{U_{r}}\right],\left[\alpha_{L_{r+1}}, \alpha_{U_{r+1}}\right], \ldots,\left[\alpha_{L_{n}}, \alpha_{U_{n}}\right]\right\}$ where $n>r ; r, n \in N$ (natural numbers) and $\left[\alpha_{L_{i}}, \alpha_{U_{i}}\right]$ 's 
are all interval-valued intuitionistic fuzzy vectors for all $i \in\{1,2, \ldots, n\}$; that is $\left[\alpha_{L_{i}}, \alpha_{U_{i}}\right] \in \widetilde{V}$. Also since $A$ is linearly dependent, there exists a vector $\left[\alpha_{L_{j}}, \alpha_{U_{j}}\right] \in A$ such that

$$
\begin{aligned}
{\left[\alpha_{L_{j}}, \alpha_{U_{j}}\right]=} & {\left[x_{L_{1}}, x_{U_{1}}\right] \cdot\left[\alpha_{L_{1}}, \alpha_{U_{1}}\right]+\left[x_{L_{2}}, x_{U_{2}}\right] \cdot\left[\alpha_{L_{2}}, \alpha_{U_{2}}\right]+\cdots } \\
& +\left[x_{L_{j-1}}, x_{U_{j-1}}\right] \cdot\left[\alpha_{L_{j-1}}, \alpha_{U_{j-1}}\right]+\left[x_{L_{j+1}}, x_{U_{j+1}}\right] \cdot\left[\alpha_{L_{j+1}}, \alpha_{U_{j+1}}\right]+\cdots \\
& +\left[x_{L_{r}}, x_{U_{r}}\right] \cdot\left[\alpha_{L_{r}}, \alpha_{U_{r}}\right]
\end{aligned}
$$

where $\left[x_{L_{i}}, x_{U_{i}}\right] \in F$ for all $i \in\{1,2, \ldots, n\}$ This implies that,

$$
\begin{aligned}
{\left[\alpha_{L_{j}}, \alpha_{U_{j}}\right]=} & {\left[x_{L_{1}}, x_{U_{1}}\right] \cdot\left[\alpha_{L_{1}}, \alpha_{U_{1}}\right]+\left[x_{L_{2}}, x_{U_{2}}\right] \cdot\left[\alpha_{L_{2}}, \alpha_{U_{2}}\right]+\ldots } \\
& +\left[x_{L_{j-1}}, x_{U_{j-1}}\right] \cdot\left[\alpha_{L_{j-1}}, \alpha_{U_{j-1}}\right]+\left[x_{L_{j+1}}, x_{U_{j+1}}\right] \cdot\left[\alpha_{L_{j+1}}, \alpha_{U_{j+1}}\right]+\ldots \\
& +\left[x_{L_{r}}, x_{U_{r}}\right] \cdot\left[\alpha_{L_{r}}, \alpha_{U_{r}}\right]+\left[\phi_{L_{r+1}}, \phi_{U_{r+1}}\right] \cdot\left[\alpha_{L_{r+1}}, \alpha_{U_{r+1}}\right]+\ldots \\
& +\left[\phi_{L_{n}}, \phi_{U_{n}}\right] \cdot\left[\alpha_{L_{n}}, \alpha_{U_{n}}\right] .
\end{aligned}
$$

Hence $B$ is linearly dependent.

To prove (3): Given that $A \subset B$ and $B$ is linearly independent, then $A$ is also linearly independent. Assume that $A$ is linearly dependent. Using the above proof, we get that $B$ is linearly dependent. This is a contradiction by our assumption.

Hence $A$ is linearly independent.

Definition 3.10. IVIF Standard Basis: An IVIF basis $\mathscr{B}$ over the interval-valued intuitionistic fuzzy algebra $F$ is an IVIF standard basis if $\left[\alpha_{L_{i}}, \alpha_{U_{i}}\right]=\sum\left[x_{L_{i j}}, x_{U_{i j}}\right]\left[\alpha_{L_{j}}, \alpha_{U_{j}}\right]$ for $\left[\alpha_{L_{i}}, \alpha_{U_{i}}\right]$, $\left[\alpha_{L_{j}}, \alpha_{U_{j}}\right] \in \mathscr{B}$ and $\left[x_{L_{i j}}, x_{U_{i j}}\right] \in F$

Theorem 3.11. Any finitely generated IVIF subspace over F has a unique IVIF standard basis.

Proof. Assume that $\mathscr{B}$ and $\mathscr{B}^{\prime}$ are two interval-valued intuitionistic fuzzy bases with $|\mathscr{B}|=\left|\mathscr{B}^{\prime}\right|$. Since $\mathscr{B}^{\prime}$ is an IVIF basis, each element of $\mathscr{B}$ can be expressed as a linear combination of the element of $\mathscr{B}^{\prime}$.

Therefore, each element $\left[\beta_{L_{i}}, \beta_{U_{i}}\right]$ of $\mathscr{B}$ must be multiple of some element $\left[\beta_{L_{i}}^{\prime}, \beta_{U_{j}}^{\prime}\right] \in \mathscr{B}^{\prime}$. Thus $\left[\beta_{L_{i}}, \beta_{U_{i}}\right] \leq\left[\beta_{L_{j}}^{\prime}, \beta_{U_{j}}^{\prime}\right]$, similarly,

$$
\begin{aligned}
& {\left[\beta_{L_{j}}^{\prime}, \beta_{U_{j}}^{\prime}\right] \leq\left[\beta_{L_{i}}, \beta_{U_{i}}\right] } \\
\Rightarrow & {\left[\beta_{L_{i}}, \beta_{U_{i}}\right]=\left[\beta_{L_{j}}^{\prime}, \beta_{U_{j}}^{\prime}\right], }
\end{aligned}
$$

also we have $|\mathscr{B}|=\left|\mathscr{B}^{\prime}\right|$. Thus, $\mathscr{B}=\mathscr{B}^{\prime}$.

Hence the interval-valued intuitionistic fuzzy basis is unique.

Theorem 3.12. Over the interval-valued intuitionistic fuzzy algebra $F$, any two IVIF bases for a finitely generated IVIF subspace of IVIFVS have the same cardinality.

Proof. Let $\widetilde{S}$ be the set of IVIF vectors, each of whose entries is equal to some entry of an IVIF vector of any finite IVIF basis $\mathscr{B}$. This implies that $\widetilde{S}$ is a finite set. 
Case (1): $\mathscr{B}$ is not an IVIF standard basis.

$\Rightarrow\left[\beta_{L_{i}}, \beta_{U_{i}}\right]=\sum\left[x_{L_{i j}}, x_{U_{i j}}\right]\left[\beta_{L_{j}}, \beta_{U_{j}}\right]$ for some $\left[\beta_{L_{i}}, \beta_{U_{i}}\right] \in \mathscr{B}$ and $\left[x_{L_{i j}}, x_{U_{i j}}\right] \in F$ with $\left[\beta_{L_{i}}, \beta_{U_{i}}\right] \neq\left[x_{L_{i i}}, x_{U_{i i}}\right]\left[\beta_{L_{i}}, \beta_{U_{i}}\right]$. That is, $\left[\beta_{L_{i}}, \beta_{U_{i}}\right] \neq \min \left[x_{L_{i i}}, x_{U_{i i}}\right]\left[\beta_{L_{i}}, \beta_{U_{i}}\right]$. Therefore, $\left[x_{L_{i i}}, x_{U_{i i}}\right]\left[\beta_{L_{i}}, \beta_{U_{i}}\right]<\left[\beta_{L_{i}}, \beta_{U_{i}}\right]$. Let $\mathscr{B}_{1}$ be the set obtained from $\mathscr{B}$ by replacing $\left[\beta_{L_{i}}, \beta_{U_{i}}\right]$ by $\left[x_{L_{i i}}, x_{U_{i i}}\right]\left[\beta_{L_{i}}, \beta_{U_{i}}\right]$. Then

$$
\begin{gathered}
|\mathscr{B}|=\left|\mathscr{B}_{1}\right| \\
\Rightarrow\langle\mathscr{B}\rangle=\left\langle\mathscr{B}_{1}\right\rangle .
\end{gathered}
$$

Also $\mathscr{B}_{1}$ is minimal set and all the IVIF vectors of $\mathscr{B}_{1}$ becomes an IVIF standard basis, then $\mathscr{B}_{1}$ is the required IVIF standard basis with the same cardinality as $\mathscr{B}$.

If $\mathscr{B}_{1}$ is not IVIF standard basis, then repeat the process of replacing $\mathscr{B}_{1}$ by an IVIF basis $\mathscr{B}_{2}$ and proceed.

Therefore, after replacing IVIF bases of the form $\mathscr{B}$ by the form $\mathscr{B}_{i}$, the process must be terminated after some finite number of steps, which happen only if we obtained an IVIF standard basis $\mathscr{B}_{i}$ with the same cardinality as $\mathscr{B}$. This proves that, for any finite IVIF basis, there exists an IVIF standard basis with the same cardinality.

Case (2): Let $\mathscr{B}$ be an IVIF standard basis. Also, if possible, let $\mathscr{B}_{1}$ be an IVIF basis of $\widetilde{S}$ such that $|\mathscr{B}| \neq\left|\mathscr{B}_{1}\right|$, then either $\left|\mathscr{B}_{1}\right|>|\mathscr{B}|$ or $\left|\mathscr{B}_{1}\right|<|\mathscr{B}|$.

Now $\left|\mathscr{B}_{1}\right|>|\mathscr{B}|$ contradicts the definition of IVIF basis that $\mathscr{B}_{1}$ is a minimal spanning set of $\widetilde{S}$. Also if $\left|\mathscr{B}_{1}\right|<|\mathscr{B}|$, then there exists an IVIF standard basis, by using Case (1), the cardinality of $\mathscr{B}$ is equal to $\mathscr{B}_{1}$. This is a contradiction that $\mathscr{B}$ is an unique IVIF standard basis.

Hence $|\mathscr{B}|=\left|\mathscr{B}_{1}\right|$.

Theorem 3.13. Let $\widetilde{S}$ be a finitely generated IVIF subspace of $\widetilde{V_{n}}$ and let $\left\{\left[e_{L_{1}}, e_{U_{1}}\right], \ldots,\left[e_{L_{n}}, e_{U_{n}}\right]\right\}$ be the IVIF standard basis for $\widetilde{S}$. Then, for any IVIF vector $\left[\alpha_{L}, \alpha_{U}\right] \in \widetilde{V_{n}}$ can be expressed uniquely as a linear combination of IVIF vectors of the IVIF standard basis.

Proof. Let $\left\{\left[e_{L_{1}}, e_{U_{1}}\right], \ldots,\left[e_{L_{n}}, e_{U_{n}}\right]\right\}$ be the IVIF standard basis for $\widetilde{S}$ and $\left[\alpha_{L}, \alpha_{U}\right]$ be any IVIF vector of $\widetilde{V_{n}}$. Consider, $\left[\alpha_{L}, \alpha_{U}\right]=\sum_{k=1}^{n}\left[x_{L_{j}}, x_{U_{j}}\right]\left[e_{L_{j}}, e_{U_{j}}\right]$, where $\left[x_{L_{j}}, x_{U_{j}}\right] \in F$.

In this expression, the co-efficients $\left[x_{L_{j}}, x_{U_{j}}\right]$ 's are not unique.

If we write this in the matrix form as

$$
\left[\alpha_{L}, \alpha_{U}\right]=\left(\left[x_{L_{1}}, x_{U_{1}}\right],\left[x_{L_{2}}, x_{U_{2}}\right], \ldots,\left[x_{L_{n}}, x_{U_{n}}\right]\right) . E,
$$

where $E$ is the matrix whose rows are the IVIF basis vectors, then $\left[\alpha_{L}, \alpha_{U}\right]=\left[p_{L}, p_{U}\right] \cdot E$ has a solution $\left[p_{L}, p_{U}\right]=\left(\left[x_{L_{1}}, x_{U_{1}}\right],\left[x_{L_{2}}, x_{U_{2}}\right], \ldots,\left[x_{L_{n}}, x_{U_{n}}\right]\right)$.

Also it can be shown that this equation has a unique maximal solution, say, ([$\left.p_{L_{1}}, p_{U_{1}}\right],\left[p_{L_{2}}, p_{U_{2}}\right]$, $\left.\ldots,\left[p_{L_{n}}, p_{U_{n}}\right]\right)$. Then $\left[\alpha_{L}, \alpha_{U}\right]=\sum_{j=1}^{n}\left[p_{L_{j}}, p_{U_{j}}\right]\left[e_{L_{j}}, e_{U_{j}}\right]$ with $\left[p_{L_{j}}, p_{U_{j}}\right] \in F$ is the unique representation of the IVIF vector $\left[\alpha_{L}, \alpha_{U}\right]$.

Theorem 3.14. Let $\widetilde{S}$ be an IVIF vector space over $F$, and a linear IVIF span of the IVIF vectors $\left[\alpha_{L_{1}}, \alpha_{U_{1}}\right], \ldots,\left[\alpha_{L_{n}}, \alpha_{U_{n}}\right]$. If some $\left[\alpha_{L_{i}}, \alpha_{U_{i}}\right]$ is a linear combination of $\left[\alpha_{L_{1}}, \alpha_{U_{1}}\right],\left[\alpha_{L_{2}}, \alpha_{U_{2}}\right], \ldots$, $\left[\alpha_{L_{i-1}}, \alpha_{U_{i-1}}\right],\left[\alpha_{L_{i+1}}, \alpha_{U_{i+1}}\right], \ldots,\left[\alpha_{L_{n}}, \alpha_{U_{n}}\right]$ then the IVIF vectors $\left[\alpha_{L_{1}}, \alpha_{U_{1}}\right], \ldots,\left[\alpha_{L_{n}}, \alpha_{U_{n}}\right]$ also spans $\widetilde{S}$. 
Proof. Let $\widetilde{W}=\left\{\left[\alpha_{L_{1}}, \alpha_{U_{1}}\right], \ldots,\left[\alpha_{L_{n}}, \alpha_{U_{n}}\right]\right\}$ such that $\widetilde{S}=\langle\widetilde{W}\rangle$.

Since $\left[\alpha_{L_{i}}, \alpha_{U_{i}}\right]$ is a linear combination of $\left[\alpha_{L_{1}}, \alpha_{U_{1}}\right],\left[\alpha_{L_{2}}, \alpha_{U_{2}}\right], \ldots,\left[\alpha_{L_{i-1}}, \alpha_{U_{i-1}}\right],\left[\alpha_{L_{i+1}}\right.$, $\left.\alpha_{U_{i+1}}\right], \ldots,\left[\alpha_{L_{n}}, \alpha_{U_{n}}\right]$, then there exist $\left[x_{L_{j}}, x_{U_{j}}\right]$ 's for $j \in\{1,2, \ldots, i-1, i+1, \ldots, n\}$ and $\left[x_{L_{j}}, x_{U_{j}}\right] \in F$ such that $\left[\alpha_{L_{i}}, \alpha_{U_{i}}\right]=\sum_{j=1, j \neq i}^{n}\left[x_{L_{j}}, x_{U_{j}}\right]\left[\alpha_{L_{j}}, \alpha_{U_{j}}\right]$.

Since $\widetilde{S}=\langle\widetilde{W}\rangle$, any vector $\left[\beta_{L}, \beta_{U}\right] \in \widetilde{S}$ can be expressed as:

$$
\begin{aligned}
{\left[\beta_{L}, \beta_{U}\right] } & =\left[y_{L_{1}}, y_{U_{1}}\right]\left[\alpha_{L_{1}}, \alpha_{U_{1}}\right]+\left[y_{L_{2}}, y_{U_{2}}\right]\left[\alpha_{L_{2}}, \alpha_{U_{2}}\right]+\ldots \\
& +\left[y_{L_{i-1}}, y_{U_{i-1}}\right]\left[\alpha_{L_{i-1}}, \alpha_{U_{i-1}}\right]+\left[y_{L_{i+1}}, y_{U_{i+1}}\right]\left[\alpha_{L_{i+1}}, \alpha_{U_{i+1}}\right]+\ldots \\
& +\left[y_{L_{n}}, y_{U_{n}}\right]\left[\alpha_{L_{n}}, \alpha_{U_{n}}\right] \\
& =\sum_{j=1, j \neq i}^{n}\left[\left[y_{L_{j}}, y_{U_{j}}\right]\left[\alpha_{L_{j}}, \alpha_{U_{j}}\right]+\left[y_{L_{i}}, y_{U_{i}}\right]\left[\alpha_{L_{i}}, \alpha_{U_{i}}\right]\right. \\
& =\sum_{j=1, j \neq i}^{n}\left[\left[y_{L_{j}}, y_{U_{j}}\right]\left[\alpha_{L_{j}}, \alpha_{U_{j}}\right]+\left[y_{L_{i}}, y_{U_{i}}\right] \sum_{j=1, j \neq i}^{n}\left[x_{L_{j}}, x_{U_{j}}\right]\left[\alpha_{L_{j}}, \alpha_{U_{j}}\right]\right. \\
& =\sum_{j=1, j \neq i}^{n}\left[x_{L_{j}}^{\prime}, x_{U_{j}}^{\prime}\right]\left[\alpha_{L_{j}}, \alpha_{U_{j}}\right],
\end{aligned}
$$

where $\left[x_{L_{j}}^{\prime}, x_{U_{j}}^{\prime}\right]=\left[y_{L_{j}}, y_{U_{j}}\right]+\left[y_{L_{i}}, y_{U_{i}}\right]\left[x_{L_{j}}, x_{U_{j}}\right]$ for $j \in\{1,2, \ldots, i-1, i+1, \ldots, n\}$ are elements in $F$.

Since $\left[\beta_{L}, \beta_{U}\right]$ is an arbitrary vector in $\widetilde{S}$, we have $\widetilde{S}=\left\langle\widetilde{W}-\left[\alpha_{L_{i}}, \alpha_{U_{i}}\right]\right\rangle$. Thus, the vectors $\left[\alpha_{L_{1}}, \alpha_{U_{1}}\right],\left[\alpha_{L_{2}}, \alpha_{U_{2}}\right], \ldots,\left[\alpha_{L_{i-1}}, \alpha_{U_{i-1}}\right],\left[\alpha_{L_{i+1}}, \alpha_{U_{i+1}}\right], \ldots,\left[\alpha_{L_{n}}, \alpha_{U_{n}}\right]$ spans $\widetilde{S}$.

Definition 3.15. Dimension of $\widetilde{S}$ : The dimension of the finitely generated subspace $\widetilde{S}$ of an IVIF vector space $\widetilde{V_{n}}$ over the interval-valued intuitionistic fuzzy algebra $F$ denoted by $\operatorname{dim}(\widetilde{S})$ is defined to be the cardinality of the IVIF standard basis of $\widetilde{S}$.

Example 3.16. The set $\{(([1,1],[0,0]),([0,0],[1,1])),(([0,0],[1,1]),([1,1],[0,0]))\}$ forms the basis for $\widetilde{V}_{2}$. Thus $\operatorname{dim}\left(\widetilde{V}_{2}\right)=2$.

Theorem 3.17. Let $\widetilde{S}$ be an IVIF vector space over $F$ of dimension $n$ and let $\left[\alpha_{L_{1}}, \alpha_{U_{1}}\right],\left[\alpha_{L_{2}}, \alpha_{U_{2}}\right]$, $\ldots,\left[\alpha_{L_{m}}, \alpha_{U m}\right],(m<n)$ be linearly independent IVIF vectors in $\widetilde{S}$. Then, there exists a basis for $\widetilde{S}$, containing $\left[\alpha_{L_{1}}, \alpha_{U_{1}}\right],\left[\alpha_{L_{2}}, \alpha_{U_{2}}\right], \ldots,\left[\alpha_{L_{m}}, \alpha_{U_{m}}\right]$.

Proof. Let $\left[\beta_{L_{1}}, \beta_{U_{1}}\right],\left[\beta_{L_{2}}, \beta_{U_{2}}\right], \ldots,\left[\beta_{L_{n}}, \beta_{U_{n}}\right]$ be the unique IVIF standard basis for $\widetilde{S}$. Then the set $\widetilde{W}=\left\{\left[\alpha_{L_{1}}, \alpha_{U_{1}}\right],\left[\alpha_{L_{2}}, \alpha_{U_{2}}\right], \ldots,\left[\alpha_{L_{m}}, \alpha_{U_{m}}\right],\left[\beta_{L_{1}}, \beta_{U_{1}}\right],\left[\beta_{L_{2}}, \beta_{U_{2}}\right], \ldots,\left[\beta_{L_{n}}, \beta_{U_{n}}\right]\right\}$

is a linearly dependent subset of $\widetilde{S}$.

Therefore, $\left[\beta_{L_{i}}, \beta_{U_{i}}\right]$ for some $i \in\{1,2, \ldots, n\}$ is a linear combination of the vectors in $\widetilde{W}$.

Since $\widetilde{S}$ is a linear span of $\widetilde{W}$. By Theorem $3.13, \widetilde{W}-\left\{\left[\beta_{L_{i}}, \beta_{U_{i}}\right]\right\}$ also spans $\widetilde{S}$.

If the set $\widetilde{W}=\left\{\left[\beta_{L_{i}}, \beta_{U_{i}}\right]\right\}$ is a minimal set, then we have a basis for $\widetilde{S}$ as required. Otherwise, we continue the process upto $m$-th iteration until we get a basis containing $\left[\alpha_{L_{1}}, \alpha_{U_{1}}\right],\left[\alpha_{L_{2}}, \alpha_{U_{2}}\right]$, $\ldots,\left[\alpha_{L_{m}}, \alpha_{U_{m}}\right]$.

Theorem 3.18. Any set of $(n+1)$ vectors in $\widetilde{V_{n}}$ is linearly dependent.

Proof. If the set $(n+1)$ vectors in $\widetilde{V_{n}}$ is linearly independent, then by Theorem 3.13 , we can find a basis for $\widetilde{V_{n}}$ containing the set. That is $(n+1)$ vectors. Which is a contradiction by our assumption. Every IVIF basis for $\widetilde{V_{n}}$ must contain $n$ vectors. Thus any set of $(n+1)$ vectors in $\widetilde{V_{n}}$ is linearly dependent. 


\section{References}

[1] Atanassov, K. T. Intuitionistic Fuzzy Sets, VII ITKR Session, Sofia, 20-23 June 1983 (Deposed in Centr. Sci.-Techn. Library of the Bulg. Acad. of Sci., 1697/84) (in Bulgarian). Reprinted: Int. J. Bioautomation, 2016, 20(S1), S1-S6.

[2] Atanassov, K. T. \& Gargov, G. (1989). Interval-valued intuitionistic fuzzy sets, Fuzzy Sets and Systems, 31, 343-349.

[3] Chiney, M. \& Samanta, S. K. (2017). Intuitionistic fuzzy basis of an intuitionistic fuzzy vector space, Notes on Intuitionistic Fuzzy Sets, 23 (4), 62-74.

[4] Gehrke, M., Walker, C., \& Walker, E., (2001). Some basic theory of interval-valued fuzzy sets, Transactions of the Joint IFSA World Congress and 20-th NAFIPS International Conference, July 25-28, 2001, Vancouver, British Columbia, 1332-1336.

[5] Hosseini, S. B., O’Regan, D. \& Saadati, R. (2007). Some results on intuitionistic fuzzy spaces, Iranian Journal of Fuzzy Systems, 4 (1), 53-64.

[6] Huang, C. E. \& Shi, F. G. (2012). On the fuzzy dimensions of fuzzy vector spaces, Iranian Journal of Fuzzy Systems, 9 (4), 141-150.

[7] Katsaras, A. K. \& Liu, D. B. (1997). Fuzzy vector spaces and fuzzy topological vector spaces, Journal of Mathematical Analysis and Applications, 58, 135-146.

[8] Kumar, R. (1992). Fuzzy vector spaces and fuzzy cosets, Fuzzy Sets and Systems, 45, 109116.

[9] Lubczonok, P. (1990). Fuzzy vector spaces, Fuzzy Sets and Systems, 38, 329-343.

[10] Malik, D. S. \& Mordeson, J. N. (1991). Fuzzy vector spaces, Inform. Sci., 55 (1-3), 271-281.

[11] Mondal, S. (2012). Interval-valued fuzzy vector space, Annals of Pure and Applied Mathematics, 2 (1), 86-95.

[12] Mordeson, J. N. (1993). Bases of fuzzy vector spaces, Inform. Sci., 67 (1-2), 87-92.

[13] Pradhan, R., \& Pal, M. (2012). Intuitionistic fuzzy linear transformations, Annals of Pure and Applied Mathematics, 1 (1), 57-68.

[14] Pradhan, R., \& Pal, M. (2014). Solvability of system of intuitionistic fuzzy linear equations, International Journal of Fuzzy Logic Systems, 4 (3), 13-24.

[15] Shi, F. G. \& Huang, C. E. (2010). Fuzzy bases and the fuzzy dimension of fuzzy vector space, Mathematical Communications, 15 (2), 303-310.

[16] Zadeh, L. A. (1965). Fuzzy sets, Information and Control, 8, 338-353. 